\title{
A Fast Detection and Recognition Algorithm with Construction of Fast Support Vector Machine based on Entropy Weight
}

\author{
$\mathrm{Zhu} \mathrm{Li}^{1}$ \\ ${ }^{1}$ Department of Computer, Xi'an University of Science and Technology, \\ $X i$ 'an 710054, China \\ E-mail: z_l_96@163.com
}

\begin{abstract}
In gesture recognition, gesture is easy to be influenced by nonlinear factors such as illumination, wrists and motion blur, etc., based on the Analysis of the SVM classification method and its effect in practical application, this paper proposes a gesture method based on the combination of nonlinear support vector machine (SVM) and Linear Discriminant Analysis, LDA (SVM+LDA). First it works out gesture recognition system constituted by multiple-surface electrode sensor, microcontroller acquisition unit, and computer; second, to get the best classification feature of image through linear discriminant analysis with LDA feature extraction; finally, to carry out classification recognition on gesture characteristic vector by applying the nearest neighbor classifier, at the same time compared with other classification algorithms to get the advantages and disadvantages of the algorithm in this paper. The experimental results show that the $S V M+L D A$ can gain higher recognition rate, so as to provide theoretical and data support for the researcher of electromyogram artificial hands control to choose the appropriate means of recognition.
\end{abstract}

Keywords: Linear discriminant analysis; Feature extraction; Recognition system; Gesture recognition; Support vector machine (SVM)

\section{Introduction}

Gesture recognition is a kind of non-contact signal recognition technology, in recent years, the appropriate means of recognition to be selected by electromyogram artificial hands control personnel has become a hot topic. Compared with contact methods such as fingerprint and iris recognition, gesture recognition has more humanized advantages. Due to its challenges existing in science and potential wide application prospect, it has become a hot spot in the field of computer vision and pattern recognition [1-5]. The effectiveness of gesture recognition, which mainly depends on the representation of features and classifier design, good features should have the characteristics of low dimension, simple classifier design, and high recognition rate. The literature [1] [2] expound the previous research results of gesture recognition roundly. Due to the feature extraction based on a single method has certain limitations, literature [3-5] put forward some methods of multiple feature extraction methods combination, can obtain better recognition results compared to the single feature extraction method. Linear Discriminant Analysis, LDA can make the pattern samples after projection have the maximum interclass distance and the minimum inner-class distance, and can effectively reduce the dimension of feature vector, but the classification performance is declined seriously after image translation, rotation and other geometry transformations [6].

In order to evaluate the operational performance of nonlinear SVM and linear LDA in the gesture recognition application of electromyogram signal, we design the sEMG gesture recognition system, and compared through experiment, and the recognition rate of two kinds of recognition algorithms under different number of electromyography 
acquisition electrodes, so as to provide theoretical and data support for the researcher of electromyogram artificial hands control to choose the appropriate means of recognition.

\section{Systematic Design}

\subsection{Recognition System Structure}

The block diagram of sEMG gesture recognition system is shown in figure 1.this system is composed of multi-surface electrode sensor, microcontroller acquisition unit, and computer. Electrode sensor is. Then microcontroller AD collecting and sends through a serial port by microcontroller computer, the computer is used to analyze and recognize surface electromyogram signal [7-8].

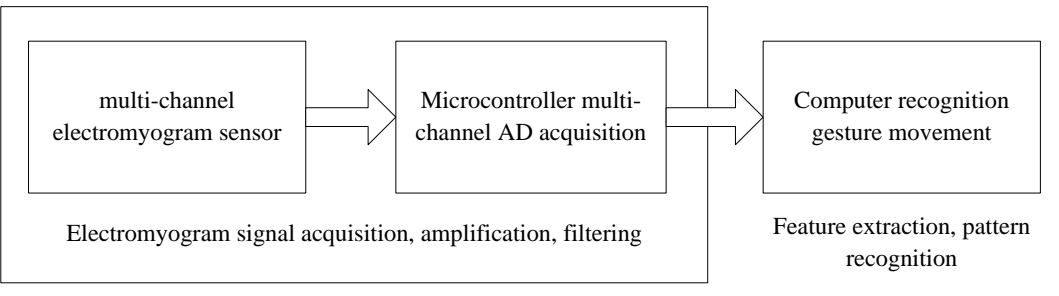

\section{Figure 1. The Block Diagram of sEMG Gesture Recognition System}

The peak-to-peak value scope of gesture signal amplitude is $0-7 \mathrm{mV}$, frequency is 0 $1000 \mathrm{~Hz}$, the maximum frequency of different electromyogrampower spectrum, usually between $40-350 \mathrm{~Hz}$.In this system, the signal is amplified by 1000 times, cut-off frequency of band-pass filter is $10-1000 \mathrm{~Hz}$. The single is sampling by microcontroller 16bit $\mathrm{AD}$ after amplification, and the sampling frequency is $1 \mathrm{KHZ}$. Finally it is sent into PC for feature extraction and pattern recognition of electromyography motion.

\subsection{Linear Discriminant Analysis (LDA)}

Linear discriminant analysis is operated by differential operation and autocorrelation matrix detection angular point, has the features as simple calculation, uniform and reasonable extracted angular point feature, quantitative extraction and stable operator[912], for the gray edge image, it carries out Harris angular point detection to generate angular point image $\mathrm{Lg}$, gesture recognition method based on LDA will change the obtained two-dimensional image feature extraction into multiple points in highdimensional space. For the sample set composed by $N$ images of $C$ people, set a total of $\omega_{1}, \omega_{2}, \ldots, \omega_{C}$ class, assumed the prior probability of $\omega_{i}(i=1,2, \ldots, C)$ class pattern is $P\left(\omega_{i}\right)$, $N_{i}(i=1,2, \ldots, C)$ is the sample number of all kinds of patterns. Then inner-class dispersion matrix $S_{w}$ and inter-class dispersion matrix $S_{b}$ can be expressed as:

$S_{w}=\sum_{i=1}^{C} P\left(\omega_{i}\right) \frac{1}{N_{i}} \sum_{j=1}^{N_{i}}\left(x_{j}^{(i)}-m_{i}\right)\left(x_{j}^{(i)}-m_{i}\right)^{T}$

$S_{b}=\sum_{i=1}^{c} P\left(\omega_{i}\right)\left(m_{i}-m\right)\left(m_{i}-m\right)^{T}$

$$
\text { Where, } \quad m_{i}=\frac{1}{N_{i}} \sum_{j=1}^{N_{i}} x_{j}^{(i)}, \quad m=\frac{1}{N} \sum_{i=1}^{C} \sum_{j=1}^{N_{i}} x_{j}^{(i)} \text {. }
$$

In order to minimize inner-class dispersion and maximize the inter-class dispersion, Fisher criterion function is as follows: 
$W^{*}=\left[w_{1}, w_{2}, \ldots, w_{k}\right]=\arg \max _{W} \frac{\left|W^{T} S_{b} W\right|}{\left|W^{T} S_{w} W\right|}$

Where, $\left\{w_{i} \mid i=1,2, \ldots, k\right\}$ is diminishing feature value set, $\left\{\lambda_{i} \mid i=1,2, \ldots, k\right\}$ is the generalized eigenvalue vector set. The optimal $W^{*}$ matrix column is corresponding to the feature vector of maximum eigenvalue of:

$S_{b} w_{i}=\lambda_{i} S_{w} w_{i}$

In order to solve $W^{*}$, by formula (4), using the irreversible ${ }^{S_{w}{ }^{-1}}$, the maximum of matrix $S_{w}$ rank is $N-C$, so the total samples in training set shall be smaller than the pixel

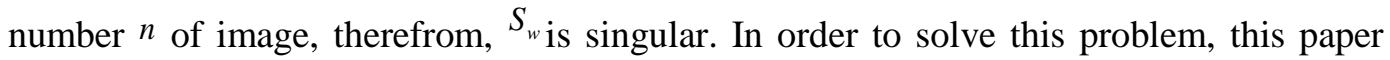
adopts SVM set LDA method, first conducts image transformation to get feature vector of $d(d<N)$ dimension, then construct gesture electromyogram signal by feature vector, so as to realize LDA transformation of feature vector.

\subsection{Support Vector Machine SVM Classifier}

The design of classifier is an important part in the process of gesture recognition system design. After extracting the feature of image to be tested, you need to use the extracted feature vector to calculate and analyze the level of similarity among images, to determine the identity of the gesture in image. In general case, the most frequently-used methods of discrimination and classification are depending on pattern recognition and machine learning. Such as the nearest neighbor method, Bayesian Decision Theory, artificial neural network and support vector machine, etc.

The focus of this paper is image feature extraction algorithm, thus it selects the most basic and frequently-used distance classification as classification method. The so-called distance classification method is a kind of simple and straightforward classification method, which directly uses the region composed by feature set of all kinds of training sample points to represent each decision domain, the distance between the sample points as main scalar to measure the similarity between samples, namely the distance between two points in declaration space is more close, the similarity between two samples is much higher. The distance between samples has a variety of calculation methods, Euclidean distance is the most common distance classification method in all kinds of calculation method:

Euclidean distance is also called as $L_{2}$ normal form, which is widely used in distance measurement among vectors, its definition as follows:

$d=\sqrt{\sum_{i=0}^{k}\left(x_{i}-r_{i}\right)^{2}}$

In formula (2.32), ${ }^{x_{i}}$ denotes the ith component of input feature vector $\mathrm{x},{ }^{r_{i}}$ denotes the ith component of reference feature vector $r, k$ denotes the dimension of feature vector. Then the minimum Euclidean distance of corresponding point and inter-class is defined as:

$d=\min _{c}\left(\sqrt{\sum_{i=0}^{k}\left(x_{i}-r_{i}^{c}\right)^{2}}\right)$ 
In formula (2.33), ${ }^{r_{i}^{c}}$ denotes the ith element of the cth feature vector of reference $\min$

class, $c_{c}$ denotes acquaintance feature vector and the minimum Euclidean distance among all $\mathrm{c}$ reference feature vectors in reference class.

Based on the Euclidean distance classification, this paper applies the nearest neighbor classifier for classification and recognition of gesture feature vector, that is the sample to be tested and training sample with the nearest distance to it are defined as the same class.

\section{Experiment}

This paper plans to apply the gesture signals of five selected able-bodied persons as electromyogram acquisition objectives, 200MS electromyogram signal sequence is used for feature extraction, the electromyogram data acquisition figure as shown in Figure 2. Each measured arm does 20 times, and records the electromyogram signal. A data set of electromyogram signal is composed of sEMG data measured from multiple channels within 1000 milliseconds time. Then, the study data and categorical data generated from the measured data set. In 1000 milliseconds of sEMG data is divided into20 segments, every 200 milliseconds as the time span of feature extraction, and start to move at the interval of every 100 milliseconds, as shown in Figure 3.The first paragraph is set as learning data, all of the 20 segments as categorical data. Segmentation diagram is shown in Figure 3.

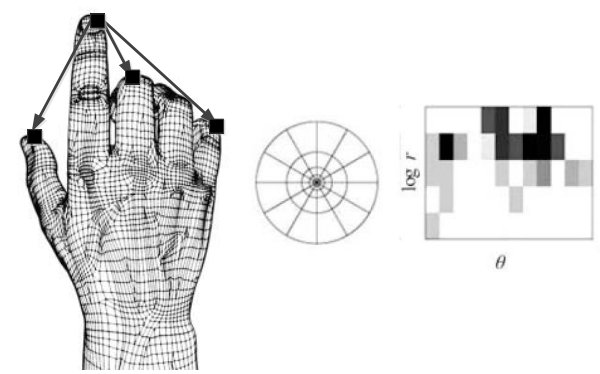

Figure 2. Data Acquisition of Electromyogram Signal

Through collecting electromyogram signal data, then using linear LDA and nonlinear SVM algorithm (using RBF kernel) for estimation, and then through sample learning, to realize the recognition and classification of LDA + SVM algorithm to gesture movement data by classification learning. In the experiment of this paper, it plans to recognize the following three movements. 1: the wrist bends downward and upward; 2: the palm makes a fist and stretches; 3: the palm upward and downward.

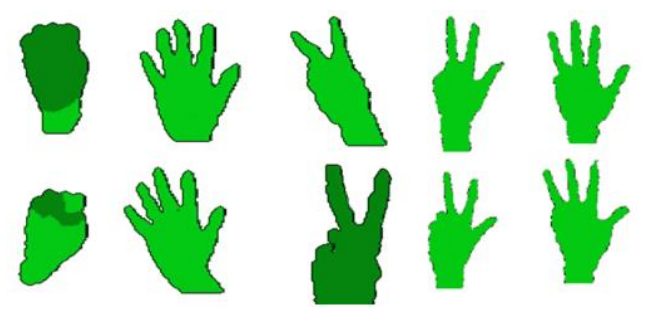

Figure 3. Segmentation Diagram of Surface Electromyogram Signal

The experimental results are shown in Table 1,2. The data indicates the relations of electrode number and the average recognition rate of two algorithms in three experimental movements (movement1, 2 and 3), respectively. Table 1 is the recognition results at the condition of different electrode number by using linear SVM + LDA 
algorithm, Table 2 is the comparison results by using nonlinear SVM algorithm and the SVM + LDA algorithm for recognition. The data in the table show that the recognition rate of SVM + LDA is higher than the recognition rate of linear SVM.

\section{Table 1. SVM and LDA Recognize the Downward and Upward Bending of Wrist}

\begin{tabular}{ccc}
\hline electrode number & C parameter & Recognition rate [\%] \\
\hline 1ch & 1.005228017 & 6.58 \\
$2 \mathrm{ch}$ & 122.9139409 & 42.87 \\
$3 \mathrm{ch}$ & 13.4209074 & 90.27 \\
$4 \mathrm{ch}$ & 247.2567827 & 92.55 \\
$5 \mathrm{ch}$ & 25.3096422 & 95.26 \\
$6 \mathrm{ch}$ & 621.6316284 & 97.61 \\
\hline
\end{tabular}

Table 2. Algorithm Performance Comparison Table

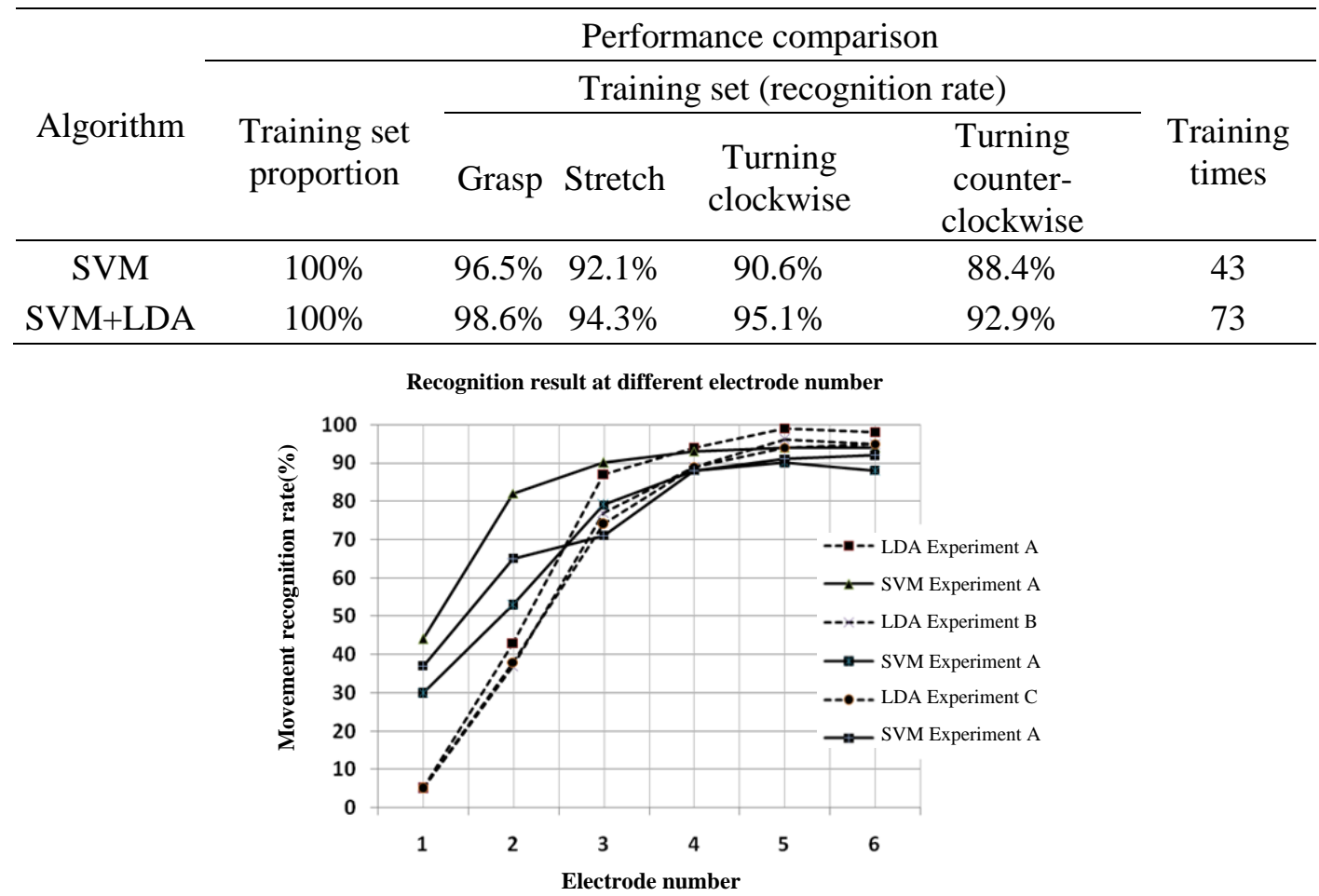

Figure 4. The Recognition Rate of LDA and SVM Algorithm at Different Electrode Number

Algorithm performance comparison is shown in Table 4, the recognition rate of using linear discriminant LDA is generally higher than that of using nonlinear SVM recognition algorithm, the high and low of recognition rate, of course, is related to algorithm and training times, in addition, it is also related to signal quality, how to reduce the noise interference will become the focus of the work in the future, the recognition rate of above-mentioned LDA and SVM algorithm at different electrode number can be drawn into a chart, as shown in Figure 4. The solid line in figure is the recognition rate of nonlinear SVM algorithm, and the dotted line is the recognition rate of LDA algorithm, we can see clearly that, when the number of electrodes is 3 , it is the point of intersection of the recognition rate of two algorithms. Linear LDA algorithm structure is simple and 
has good recognition rate, but in the case of electrode number is small, its recognition rate is largely lower than that of nonlinear SVM algorithm. This provides data support for multiple gesture movements recognition research by using two electrodes acquisition electromyogram signal accurately.

\section{Acknowledgement}

The research is supported by Education Department of Shaanxi Provincial Government Research Project (2013JK1140), Shaanxi science and technology research and development project (2014K05-37) and National Natural Science Foundation of China, Coal joint fund project (U1261114).

\section{References}

[1] Yang J., He S. and Lin Y., "Multimedia cloud transmission and storage system based on internet of things[J]", Multimedia Tools and Applications, (2015).

[2] $\mathrm{Li}$ X., $\mathrm{Lv} \mathrm{Z}$. and $\mathrm{Hu}$ J., "XEarth: A 3D GIS Platform for managing massive city information[C]",//Computational Intelligence and Virtual Environments for Measurement Systems and Applications (CIVEMSA), 2015 IEEE International Conference on. IEEE, (2015), pp. 1-6.

[3] Yang J., Chen B. and Zhou J., "A Low-Power and Portable Biomedical Device for Respiratory Monitoring with a Stable Power Source[J]”, Sensors, vol. 15, no. 8, (2015), pp. 19618-19632.

[4] Gu W., Lv Z. and Hao M., "Change detection method for remote sensing images based on an improved Markov random field[J]", Multimedia Tools and Applications, (2015), pp. 1-16.

[5] Chen Z., Huang W. and Lv Z., "Towards a face recognition method based on uncorrelated discriminant sparse preserving projection[J]", Multimedia Tools and Applications, (2015), pp. 1-15.

[6] Su T., Wang W. and Lv Z., "Rapid Delaunay triangulation for randomly distributed point cloud data using adaptive Hilbert curve[J]", Computers \& Graphics, vol. 54, (2016), pp. 65-74.

[7] G. Bao, L. Mi, Y. Geng and K. Pahlavan, "A computer vision based speed estimation technique for localizing the wireless capsule endoscope inside small intestine", 36th Annual International Conference of the IEEE Engineering in Medicine and Biology Society (EMBC), (2014) Aug.

[8] X. Song and Y. Geng, "Distributed community detection optimization algorithm for complex networks", Journal of Networks, vol. 9, no. 10, (2014) Jan., pp. 2758-2765.

[9] J. He, Y. Geng and K. Pahlavan, "Toward Accurate Human Tracking: Modeling Time-of-Arrival for Wireless Wearable Sensors in Multipath Environment", IEEE Sensor Journal, vol. 14, no. 11, pp. 39964006, (2014) Nov.

[10] Lv Z., Halawani A. and Fen S., "Touch-less Interactive Augmented Reality Game on Vision Based Wearable Device[J]”, Personal and Ubiquitous Computing, vol. 19, no. 3, (2015), pp. 551-567.

[11] Gu W., Lv Z. and Hao M., "Change detection method for remote sensing images based on an improved Markov random field[J]", Multimedia Tools and Applications, (2015), pp. 1-16.

[12] Lv Z., Tek A. and Da Silva F., "Game on, science-how video game technology may help biologists tackle visualization challenges[J]", PloS one, vol. 8, no. 3, (2013), pp. 57990.

[13] Chen Z., Huang W. and Lv Z., "Towards a face recognition method based on uncorrelated discriminant sparse preserving projection[J]", Multimedia Tools and Applications, (2015), pp. 1-15.

[14] Jiang D., Ying X. and Han Y., "Collaborative multi-hop routing in cognitive wireless networks[J]", Wireless Personal Communications, (2015), pp. 1-23.

[15] Yang J., He S. and Lin Y., "Multimedia cloud transmission and storage system based on internet of things[J]", Multimedia Tools and Applications, (2015), pp. 1-16.

[16] Lv Z., Yin T. and Han Y., "WebVR—— web virtual reality engine based on P2P network[J]", Journal of Networks, vol. 6, no. 7, (2011), pp. 990-998.

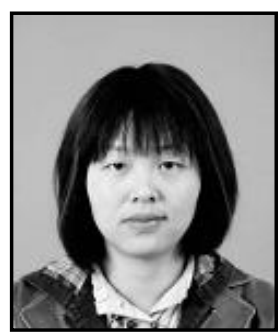

Zhu Li, She received her Master's degree in software engineering from Xi'an University of Science and Technology, in Xi'an, China. She is currently a lecturer in Xi'an University of Science and Technology. Her research interest is mainly in the area of Computer Software, Pattern recognition and machine learning. She has published several research papers in scholarly journals in the above research areas and has participated in several books. 